\title{
In silico screening method for non-responders to cardiac resynchronization therapy in patients with heart failure: a pilot study
}

\author{
Minki Hwang ${ }^{1}$, Jae-Sun Uhm² ${ }^{*}$, Min Cheol Park ${ }^{3}$, Eun Bo Shim ${ }^{1,3}$, Chan Joo Lee ${ }^{4}$, Jaewon Oh ${ }^{4}$, Hee Tae Yu ${ }^{4}$,
} Tae-Hoon Kim ${ }^{4}$, Boyoung Joung ${ }^{4}$, Hui-Nam Pak ${ }^{4}$, Seok-Min Kang ${ }^{4}$ and Moon-Hyoung Lee ${ }^{4}$

\begin{abstract}
Background: Cardiac resynchronization therapy (CRT) is an effective treatment option for patients with heart failure (HF) and left ventricular (LV) dyssynchrony. However, the problem of some patients not responding to CRT remains unresolved. This study aimed to propose a novel in silico method for CRT simulation.

Methods: Three-dimensional heart geometry was constructed from computed tomography images. The finite element method was used to elucidate the electric wave propagation in the heart. The electric excitation and mechanical contraction were coupled with vascular hemodynamics by the lumped parameter model. The model parameters for three-dimensional (3D) heart and vascular mechanics were estimated by matching computed variables with measured physiological parameters. CRT effects were simulated in a patient with HF and left bundle branch block (LBBB). LV end-diastolic (LVEDV) and end-systolic volumes (LVESV), LV ejection fraction (LVEF), and CRT responsiveness measured from the in silico simulation model were compared with those from clinical observation. A CRT responder was defined as absolute increase in $\mathrm{LVEF} \geq 5 \%$ or relative increase in $\mathrm{LVEF} \geq 15 \%$.

Results: A 68-year-old female with nonischemic HF and LBBB was retrospectively included. The in silico CRT simulation modeling revealed that changes in LVEDV, LVESV, and LVEF by CRT were from 174 to $173 \mathrm{~mL}, 116$ to $104 \mathrm{~mL}$, and 33 to 40\%, respectively. Absolute and relative $\triangle L V E F$ were $7 \%$ and 18\%, respectively, signifying a CRT responder. In clinical observation, echocardiography showed that changes in LVEDV, LVESV, and LVEF by CRT were from 162 to $119 \mathrm{~mL}, 114$ to $69 \mathrm{~mL}$, and 29 to 42\%, respectively. Absolute and relative $\triangle \mathrm{LVESV}$ were 13\% and 31\%, respectively, also signifying a CRT responder. CRT responsiveness from the in silico CRT simulation model was concordant with that in the clinical observation.
\end{abstract}

Conclusion: This in silico CRT simulation method is a feasible technique to screen for CRT non-responders in patients with HF and LBBB.

Keywords: Cardiac resynchronization therapy, In silico modeling, Non-responder, Virtual CRT

*Correspondence: jason@yuhs.ac

${ }^{2}$ Department of Cardiology, Yongin Severance Hospital, Yonsei University College of Medicine, 363 Dongbaekjukjeon-daero, Giheung-gu, Yongin-si, Gyeonggi-do 16995, Republic of Korea

Full list of author information is available at the end of the article

\section{INTRODUCTION}

Cardiac resynchronization therapy (CRT) is one of the established treatment options for patients with heart failure (HF) and left ventricular (LV) dyssynchrony [1]. Patients with CRT benefit from the effects of electric original author(s) and the source, provide a link to the Creative Commons licence, and indicate if changes were made. The images or other third party material in this article are included in the article's Creative Commons licence, unless indicated otherwise in a credit line to the material. If material is not included in the article's Creative Commons licence and your intended use is not permitted by statutory regulation or exceeds the permitted use, you will need to obtain permission directly from the copyright holder. To view a copy of this licence, visit http://creativecommons.org/licenses/by/4.0/. 
and mechanical synchronization of their ventricular muscle and LV function is ultimately improved. However, CRT is not effective in approximately $1 / 3$ of the patients and this CRT non-responder issue has not been resolved to date $[2,3]$. Therefore, for achieving a more ubiquitous performance of CRT in patients with HF, patient-specific simulation methods for screening CRT non-responders need to be established. In view of the many difficulties associated with performing an experimental or clinical study using a living heart, computer simulation can be an alternative method to understand the physiological mechanisms of CRT. Hitherto, computer simulation of cardiac electrophysiology and mechanics has been considered a useful tool for the investigation of the pathophysiology of heart disease and for the development of novel therapeutic clinical techniques [4]. For example, a multi-scale heart modeling was developed by integrating cell, tissue, and organ scales to investigate the electrophysiological behavior of the heart [5]. However, most of the computational studies on the mechanism of heart disease have been performed not on a patient-specific heart model, but on a typical heart model. For CRT simulation, a patient-specific heart model is essential. In this study, we propose a patient-specific cardiac electromechanical model of pre- and post-CRT treatment to screen for CRT non-responders in patients with HF and left bundle branch block (LBBB).

\section{Methods}

\section{Electromechanical heart model}

To construct an integrated CRT simulation model, we combined the 3-dimensional (3D) image-based electromechanical model of a patient with LBBB with the lumped model of the circulatory system and electric pacing device [6]. A schematic diagram of the integrated model is shown in Fig. 1. To simulate the electric and mechanical behavior of the heart during cardiac tissue excitation and contraction, we used the electromechanical model that has two dynamic components, electrical and mechanical, as described previously [7]. Physiologically, as an electric wave propagates through the heart, the depolarization of each myocyte initiates the release of calcium $\left(\mathrm{Ca}^{2+}\right)$ from the stores in the sarcoplasmic reticulum, which is followed by the binding of $\mathrm{Ca}^{2+}$ to troponin $\mathrm{C}$ and cross-bridge cycling. The cross-bridge cycling forms the basis for sliding of myosin (thick) filaments relative to actin (thin) filaments and the development of active tension in the cells, resulting in contraction of the ventricles.

The electrical component of the model simulates the propagation of a wave of transmembrane potential by solving the monodomain equations on the electrical mesh as shown in Eq. 1.

$$
\frac{\partial V_{m}}{\partial t}=-\left(I_{\text {ion }}+I_{\text {app }}\right) \frac{1}{C_{m}}+\left(\nabla \cdot D \nabla V_{m}\right)
$$

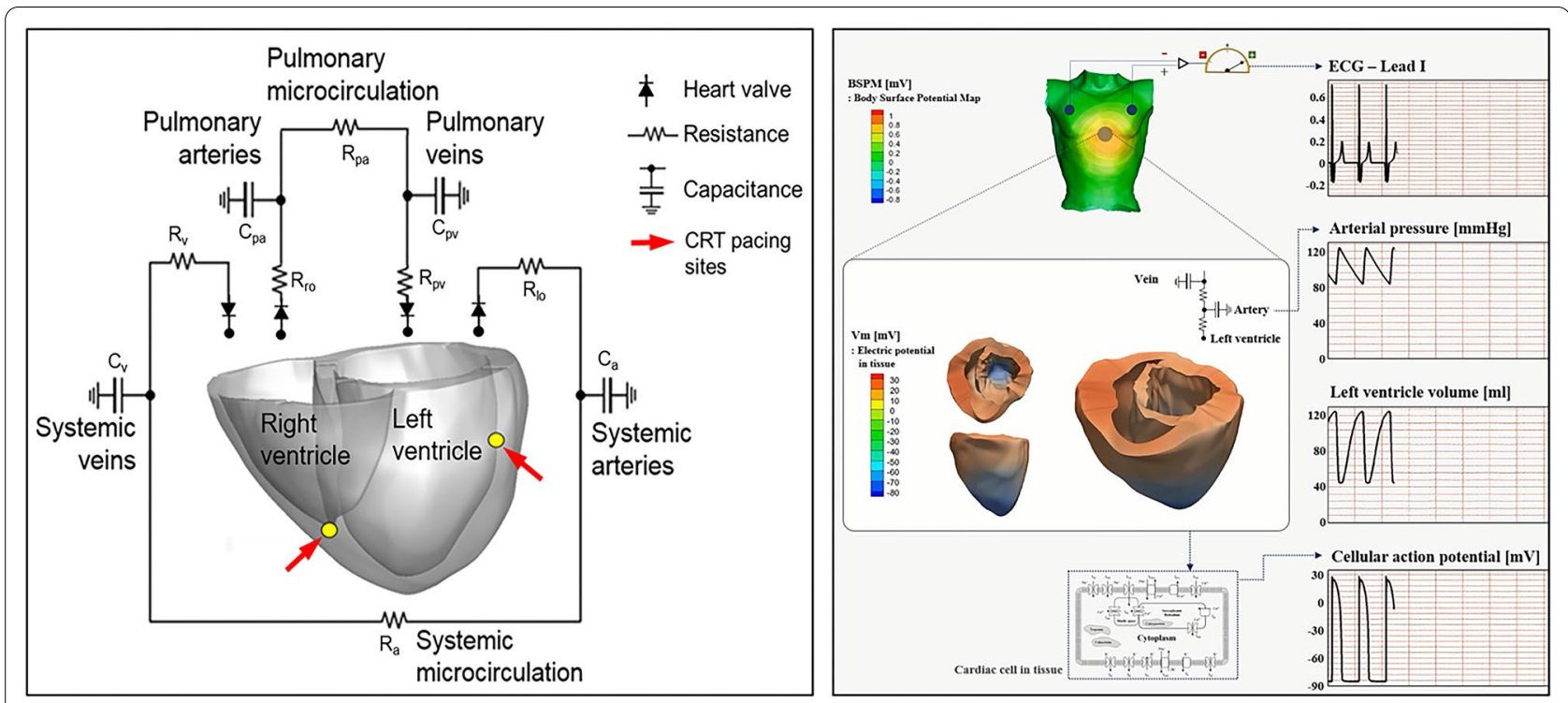

Fig. 1 The schema of the finite element ventricular electromechanical model coupled with the circulatory and CRT models. $C_{a}=$ systemic artery compliance, $C_{\mathrm{pa}}=$ pulmonary artery compliance, $C_{\mathrm{pv}}=$ pulmonary vein compliance, $C R T=$ cardiac resynchronization therapy, $C_{\mathrm{v}}=$ systemic vein compliance, ECG = electrocardiography, $R_{\mathrm{a}}=$ systemic artery resistance, $R_{\mathrm{lo}}=$ left ventricular outflow resistance, $R_{\mathrm{pa}}=$ pulmonary artery resistance, $R_{\mathrm{pv}}=$ pulmonary vein resistance, $R_{\mathrm{v}}=$ systemic vein resistance 
Here, $\mathrm{t}, V_{m}, I_{\text {ion }}, I_{\text {app }}, C_{m}$, and $D$ are time, membrane voltage, current across the cellular membrane, stimulation current, membrane capacitance, and electric diffusion coefficient, respectively. To solve the equation, we spatially discretized the equation by using the finite element method and time derivative in the equation was approximated by forward Euler method. More detailed explanation for the solution of the equation is shown in our previous paper [8].

A calcium transient serves as an input to the cell myofilament model representing the generation of active tension within each myocyte, where a set of ordinary differential equations and algebraic equations describes $\mathrm{Ca}^{2+}$ binding to troponin $\mathrm{C}$, cooperativity between the regulatory proteins, and cross-bridge cycling. Contraction of the ventricles results from the active tension generation represented by the model of myofilament dynamics. Contraction is described by the equations of passive cardiac mechanics, with the myocardium being an orthotropic, hyperelastic, and nearly incompressible material with passive properties defined by an exponential strain-energy function as in our previous paper [9]. Simultaneous solution of the myofilament model equations with those representing passive cardiac mechanics on the mechanical mesh constitutes the simulation of cardiac contraction. The interaction between the electric model and mechanical one is depicted in Fig. 2. Also circulatory dynamics represented by vascular resistances (hereafter ' $R$ ') and capacitances (hereafter ' $C$ ') was combined with the heart mechanics.

\section{Patient-specific heart model for in silico CRT simulation}

The numerical methods for solving the equations of the electromechanical model have been described previously [10]. To establish LBBB model in the heart model, the left part of the Purkinje fiber networks was blocked as shown in Fig. 3. Here, the network was from our previous work [11]. A patient-specific 3D ventricular geometry was reconstructed from the computed tomography $(\mathrm{CT})$ images by using a commercial segmentation software package (Aquarius iNtuition Version 4.4.11 TeraRecon, Inc, San Mateo, CA, USA). To estimate the parameters in the model, the clinically measured values of the physiological data were used. The procedure for the estimation is as follows:

Step 1. Measure the physiological data of a patient in pre-CRT state: systolic (SBP) and diastolic blood pressures (DBP), heart rate (HR), and cardiac output (CO).

Step 2. Construct the ventricle model with 3D geometry from the CT images of the patient.

Step 3. Add the Purkinje fiber network to the 3D ventricular geometry.

Step 4. Assume the resistance (R) and capacitance (C) values of the vascular system.

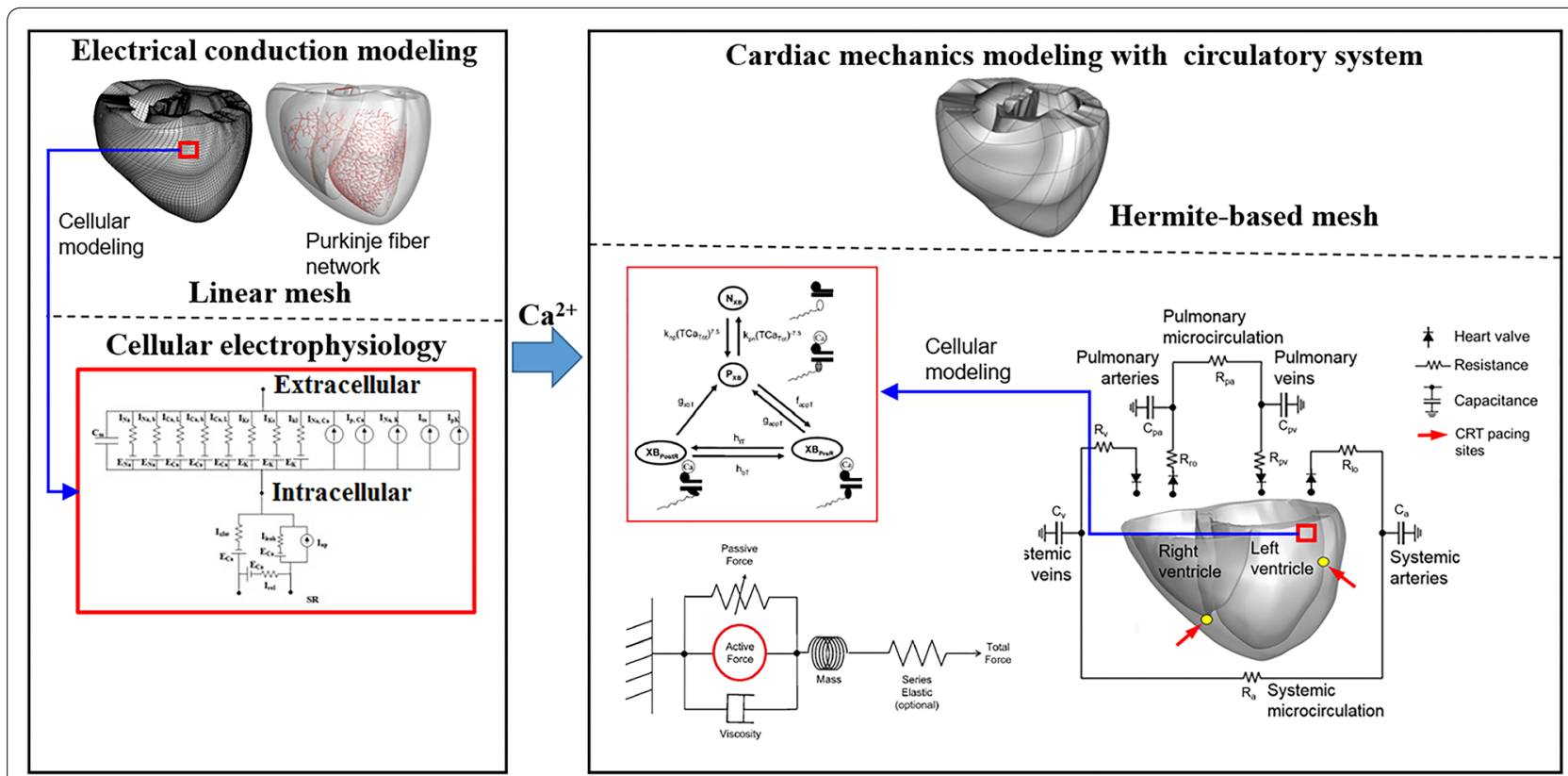

Fig. 2 The schema of the cardiac electromechanical model. Here, the cellular excitation-contraction is incorporated into the tissue mechanical model and vascular hemodynamics implemented in the lumped parameter model interacting with cardiac electromechanical contraction. $C_{\mathrm{a}}=$ systemic artery compliance, $C_{\mathrm{pa}}=$ pulmonary artery compliance, $C_{\mathrm{pv}}=$ pulmonary vein compliance, $C R T=$ cardiac resynchronization therapy, $C_{\mathrm{v}}=$ systemic vein compliance, $R_{\mathrm{a}}=$ systemic artery resistance, $R_{\mathrm{l}_{0}}=$ left ventricular outflow resistance, $R_{\mathrm{pa}}=$ pulmonary artery resistance, $R_{\mathrm{pv}}=$ pulmonary vein resistance, $R_{\mathrm{v}}=$ systemic vein resistance 

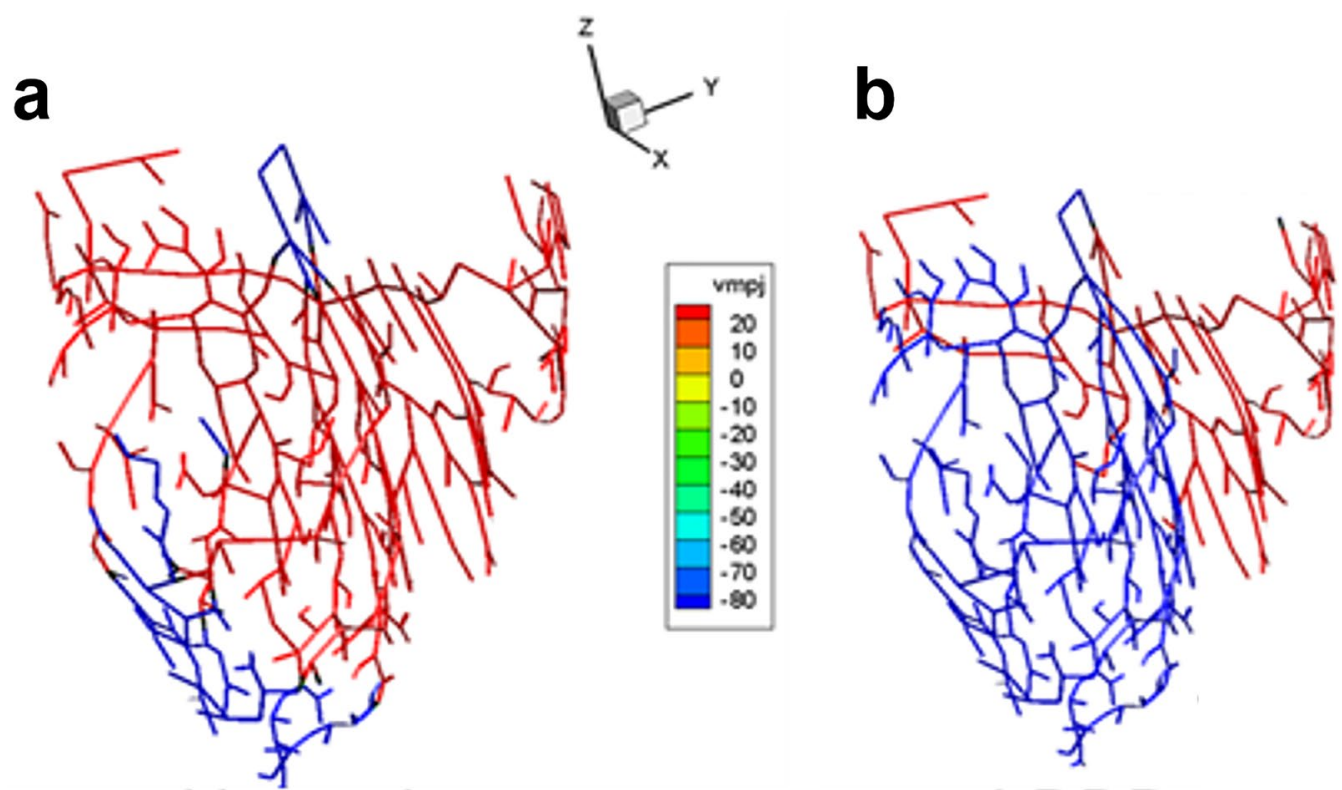

Fig. 3 Electric potentials in Purkinje fiber networks. Here, color indicates electric potentials. Red means electrically activated whereas blue means resting state. a. In normal ventricular conduction model, most parts of the ventricles activate almost simultaneously. $\mathbf{b}$. In the LBBB model, most of the left part of the Purkinje fiber network remains electrically inactivated. LBBB = left bundle branch block

Step 5. Couple the circulation model having the assumed parameters of $\mathrm{R}$ and $\mathrm{C}$ with the $3 \mathrm{D}$ ventricular mechanics model.

Step 6. Perform the simulation and obtain the computed SBP, DBP and CO of the patient.

Step 7. Calculate the errors of the computed SBP, DBP and $\mathrm{CO}$ compared with the clinically measured ones.

Step 8. If the errors are greater than 5\%, go to Step 2 with newly assumed $\mathrm{R}$ and $\mathrm{C}$ parameters. If the errors are less than 5\%, go to Step 9 since these parameters satisfy the physiological state of the patient.

Step 9. Based on the parameters, we perform CRT simulation by virtually pacing two areas on the left and right ventricular surface.

Step 10. From the computed results of CRT simulation, we determine whether the patient is a responder or non-responder.

Using the algorithm, we can match the model parameters in the simulation with clinically measured physiological properties. Then, CRT simulations based on the parameters are performed to assess whether the patient is a non-responder to CRT.

\section{Comparison of changes in LV volumes in the in silico CRT simulation model with those from clinical observation}

The study protocol was approved by the institutional ethics board of Severance Hospital, Yonsei University College of Medicine (IRB number: 1-2019-0077 and 1-2020-0008). A 68-year-old female with nonischemic HF with LBBB was retrospectively included (Table 1). Electrocardiography (ECG), echocardiography, cardiac CT and magnetic resonance (MRI) were performed before implantation of CRT device. Because LV function had not improved despite optimal medical therapy for HF for 3 months, a CRT-defibrillator was implanted in this patient. Electrocardiography and echocardiography were performed 6 months after CRT for assessing CRT responsiveness. LV end-diastolic (LVEDV) and end-systolic volumes (LVESV), and LV ejection fraction (LVEF) were measured by echocardiography before and 6 months after CRT implantation. LVEF was defined as (LVEDV - LVESV)/LVEDV $\times 100$. A CRT responder was defined as absolute increase in $\mathrm{LVEF} \geq 5 \%$ and relative increase in $\mathrm{LVEF} \geq 15 \%$ at 6 months after CRT [12]. Absolute $\triangle$ LVEF was defined as $\mathrm{LVEF}_{\text {post-CRT }}-\mathrm{LVEF}_{\text {pre-CRT }}$. Relative $\triangle \mathrm{LVEF}$ was defined as $\quad\left(\mathrm{LVEF}_{\text {post-CRT }}-\mathrm{LVEF}_{\text {pre-CRT }}\right) / \mathrm{LVEF}_{\text {pre-CRT }} \times 100$. LVEDV, LVESV, LVEF, absolute and relative $\triangle \mathrm{LVEF}$, and CRT responsiveness measured from the in silico simulation model were compared with those found in clinical observation.

\section{Results}

The patient-specific CRT model was constructed from the CT images of the patient. For the validation of the LBBB model, we compared the computed ECG data with 
Table 1 Baseline characteristics, ECG, and echocardiographic data of the patient

\begin{tabular}{|c|c|c|c|c|}
\hline & \multicolumn{2}{|c|}{ Clinical observation } & \multicolumn{2}{|c|}{ In silico simulation } \\
\hline & Before CRT & After CRT & Before CRT & After CRT \\
\hline Age (years) & 68 & & & \\
\hline Sex & Female & & & \\
\hline Hypertension & No & & & \\
\hline Diabetes & No & & & \\
\hline Cause of HF & Nonischemic & & & \\
\hline $\begin{array}{l}\text { NYHA functional } \\
\text { class }\end{array}$ & $\|$ & I & & \\
\hline $\begin{array}{l}\text { Blood pressure } \\
(\mathrm{mmHg})\end{array}$ & $120 / 68$ & $118 / 55$ & $120 / 68$ & $124 / 69$ \\
\hline \multicolumn{5}{|l|}{ ECG } \\
\hline PR interval (ms) & 168 & 150 & - & - \\
\hline $\begin{array}{l}\text { QRS duration } \\
\text { (ms) }\end{array}$ & 154 & 138 & 148 & 124 \\
\hline QRS complex & LBBB & pacing & & \\
\hline \multicolumn{5}{|l|}{ Echocardiography } \\
\hline LVEDV (mL) & 162 & 119 & 174 & 173 \\
\hline LVESV (mL) & 114 & 69 & 116 & 104 \\
\hline LVEF (\%) & 29 & 42 & 33 & 40 \\
\hline $\begin{array}{l}\text { Abso- } \\
\text { lute } \triangle \operatorname{LVEF}^{*}(\%)\end{array}$ & & 13 & & 7 \\
\hline $\begin{array}{l}\text { Relative } \triangle \mathrm{LVEF}^{\mathrm{a}} \\
(\%)\end{array}$ & & 31 & & 18 \\
\hline CRT response & & Responder & & Responder \\
\hline
\end{tabular}

$C R T$ cardiac resynchronization therapy, ECG electrocardiography, $H F$ heart failure, IVCD intraventricular conduction delay, LBBB left bundle branch block, LVEDV left ventricular end-diastolic volume, $L V E F$ left ventricular ejection fraction, LVESV left ventricular end-systolic volume, NYHA New York Heart Association,

* absolute $\triangle \mathrm{LVEF}=\mathrm{LVEF}_{\text {post-CRT }}-\mathrm{LVEF}_{\text {pre-CRT }}$

${ }^{\text {a }}$ relative $\Delta L V E F=\left(\mathrm{LVEF}_{\text {post-CRT }}-\mathrm{LVEF}_{\text {pre-CRT }}\right) / \mathrm{LVEF}_{\text {pre-CRT }} \times 100$

the clinically measured values. As shown in Fig. 4, the computed ECGs show the typical pattern of LBBB [14]. The baseline characteristics, ECG, and echocardiographic data of the patients are shown in Table 1. Based on the measured SBP, DBP, and HR, we iteratively computed the $3 \mathrm{D}$ heart model to find the simulation parameters ( $\mathrm{R}$ and $\mathrm{C}$ values) that can reproduce the measured CO. Figure 5 shows an activation map of both ventricles at 0,80 , and $140 \mathrm{~ms}$ under the LBBB and CRT conditions. In the case of LBBB, the right ventricle (RV) was activated before the $\mathrm{LV}$, which shows that the model of LBBB was correctly implemented. When CRT was performed in the model, however, the RV and LV were activated almost simultaneously, which demonstrates that this patient model responded to the CRT protocol. Figure 6 shows the changes of the arterial and LV pressures as well as the LV volume with time under the LBBB and CRT conditions. CRT increased SBP from 120 to $124 \mathrm{mmHg}$ and DBP from 68 to $69 \mathrm{mmHg}$. The in silico simulation modeling

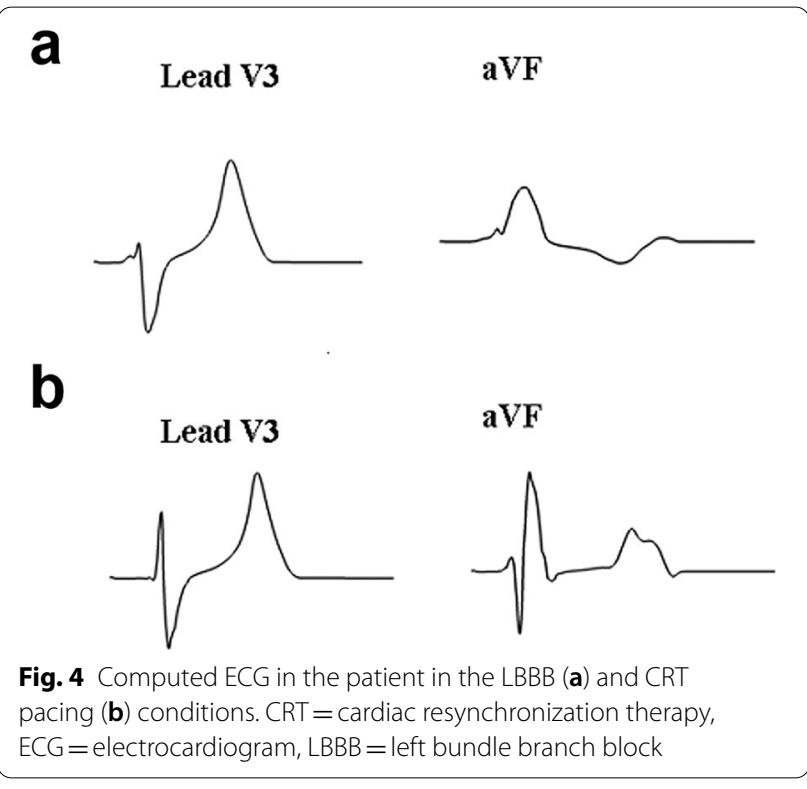

revealed that changes in LVEDV, LVESV, and LVEF by CRT were from 174 to $173 \mathrm{~mL}, 116$ to $104 \mathrm{~mL}$, and 33 to $40 \%$, respectively. From in silico modeling, absolute and relative $\triangle \mathrm{LVEF}$ were $7 \%$ and $18 \%$, respectively, and we took that to mean a CRT responder (Table 1). In clinical observation, echocardiography showed that changes in LVEDV, LVESV, and LVEF by CRT were from 162 to $119 \mathrm{~mL}, 114$ to $69 \mathrm{~mL}$, and 29 to $42 \%$, respectively. Absolute and relative $\triangle$ LVESV were $13 \%$ and $31 \%$, respectively, and that means that the patient was a CRT responder (Table 1). Therefore, CRT responsiveness from the in silico CRT simulation model was concordant with that in the clinical observation.

\section{Discussion}

Main findings

In this study, we constructed the patient-specific threedimensional electromechanical heart model with LBBB, simulated CRT on the model, and compared the cardiac parameters at pre- and post-CRT to test the applicability of the model to CRT simulation. The simulated ECGs were similar to those reported in the literature under both pre- and post-CRT conditions. The simulated electrical wave propagations in the ventricles exhibited the sequence of activation typically observed under both LBBB and CRT conditions. An improvement in LVEF was observed after CRT simulation.

\section{Prior CRT simulation models}

Because CRT is not effective in about one-third of the patients with HF and LBBB [12, 13], screening for CRT non-responders before CRT implantation is an important 
a

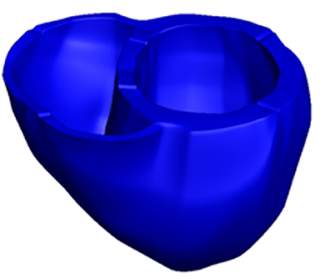

b

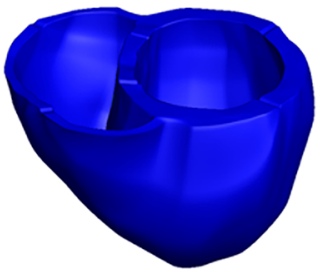

$0 \mathrm{~ms}$
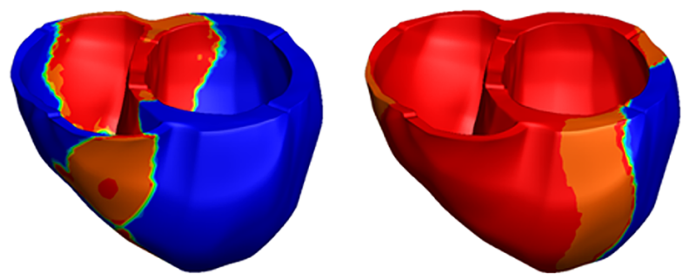

$\mathrm{V}_{\mathrm{m}}(\mathrm{mV})$

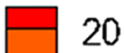

10

0

$-10$

$-20$

$-30$

$-40$

$-50$

$-60$

$-70$

$-80$

Fig. 5 The activation maps of ventricles under the LBBB (a) and CRT (b) conditions. While the RV was activated before the LV in the LBBB condition, both ventricles were activated almost simultaneously in the CRT condition. CRT = cardiac resynchronization therapy, $L B B B=$ left bundle branch block, $\mathrm{LV}=$ left ventricle, $\mathrm{RV}=$ right ventricle, $V_{\mathrm{m}}=$ membrane voltage

a

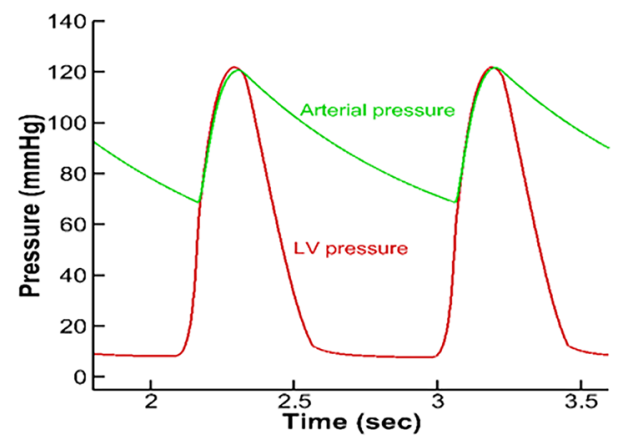

b

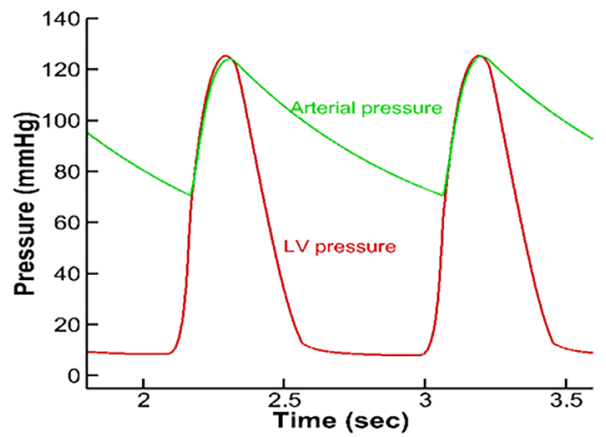

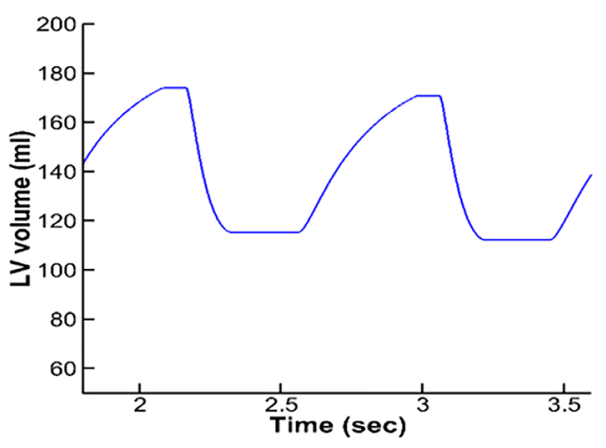

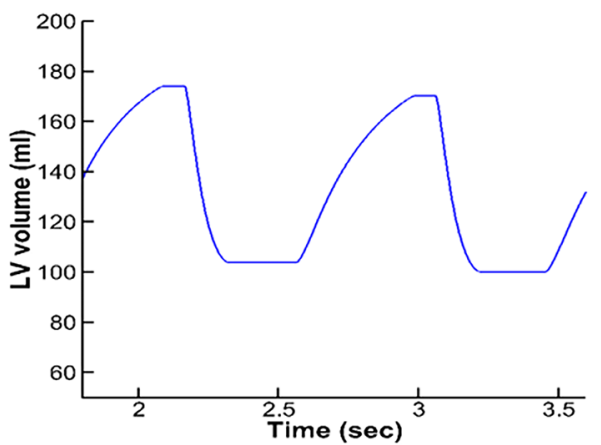

Fig. 6 Changes in pressure and LV volume with time under the LBBB (a) and CRT (b) conditions. CRT increased systolic LV pressure from 120 to $124 \mathrm{mmHg}$ and decreased LV end-systolic volume from 116 to $104 \mathrm{~mL}$, that resulted in increased LVEF from 33 to $40 \%$. CRT =cardiac resynchronization therapy, $\mathrm{LBBB}=$ left bundle branch block, $\mathrm{LV}=$ left ventricle, $\mathrm{LVEF}=$ left ventricular ejection fraction 
strategy. Computer simulation can be a useful tool for testing the effect of CRT for an individual patient before treatment. The factors affecting the efficacy of CRT include location of the LV and RV leads, atrioventricular and interventricular delay and underlying pathology [15]. Moreover, the optimal location of ventricular pacing varies in each individual patient $[16,17]$. The pacing locations and time delays can be tested using computer simulation. Isotani et al. [18] tested four different lead positions and examined the change in the maximum time derivative of ventricular pressure $\left(\% \Delta \mathrm{dP} / \mathrm{d} t_{\max }\right)$ for each location. They hypothesized that patients with insignificant gain in $\% \Delta \mathrm{dP} / \mathrm{d} t_{\max }$ even at the best lead position would be non-responders. Placing the ventricular leads on the scar region would have minimal effect on the efficacy of resynchronization. The locations and size of the scar can also be examined by constructing a 3D heart model using images from modalities such as MRI. The accuracy of the heart model for an individual patient would be critical in the interpretation of the simulation results. Okada et al. [19] constructed a heart model and simulated CRT, but they modeled the Purkinje fiber network as a thin layer on the endocardial surface. Our $3 \mathrm{D}$ electromechanical heart model has all the necessary components for individualized CRT simulation such as the Purkinje fiber network, fiber orientation, mechanical contraction/relaxation, and systemic circulation. The CRT simulation performed on one patient model in this study exhibited the possibility of utilizing this model for screening non-responders to CRT before treatment. Appropriate simulation protocols or biomarkers would have to be developed for the screening. Okada et al. [20] observed that $\mathrm{dP} / \mathrm{dt}_{\max }$ showed the best correlation with clinically observed improvement in LVEF. They also observed that the change in the duration of the QRS complex did not correlate with the clinical improvement in LVEF.

\section{Criteria of CRT responders}

It still remains controversial how and when to define CRT response. Several clinical, echocardiographic, and hemodynamic criteria for CRT response have been suggested. The echocardiographic CRT response criteria were the following: decrease in LVESV $>15 \%$, decrease in LVEDV $>15 \%$, absolute increase in $\mathrm{LVEF} \geq 5 \%$, relative increase in $L V E F \geq 15 \%$, and decrease in functional mitral regurgitation $\geq 1$ grade. "Decrease in LVESV $>15 \%$ at 6 months after CRT implantation" is generally considered as the clinically useful criterion for CRT response. However, "Decrease in LVESV $>15 \%$ " might not be appropriate as a CRT response criterion in the in silico CRT simulation model, because "decrease in LV volume" is one of the markers of long-term reverse remodeling of the $\mathrm{LV}$ and long-term reverse remodeling is not taken into account in the in silico CRT simulation model. In contrast, the in silico CRT simulation model adequately reflects the acute changes in LV contractility induced by CRT. The present results show that $\triangle \mathrm{LVEF}$ in the in silico CRT simulation model was well correlated to that in the clinical observation. Therefore, absolute and relative $\triangle \mathrm{LVEF}$ may be useful CRT response criteria in the in silico CRT simulation model.

\section{The strength of this in silico CRT simulation model}

Unlike the previous CRT simulation methods, our approach reflects the physiological measurement data in the model. Using this model, the CRT simulations for one patient were conducted, and the outcomes of the patient were analyzed. Furthermore, we demonstrated that this computational model can be used as a screening tool for CRT non-responders before CRT device implantation.

\section{Study limitations}

The individual anatomy of the Purkinje fiber networks cannot be visualized. The vascular resistance cannot be noninvasively measured. Therefore, the anatomy of the Purkinje fiber networks and vascular resistance were assumed in this in silico CRT simulation model based on the data from the general population. [11] This in silico CRT simulation model can reflect acute changes in LV volume and motion induced by CRT. Long-term reverse remodeling of the LV is not considered in this model. The present study was a pilot study and limited to only one patient. Because the present stud was retrospective, bias might be involved. Validating our model for a large number of patients is necessary for our model to be used as a screening tool for response to CRT.

\section{Conclusion}

This in silico CRT simulation method of a 3D patientspecific heart is feasible as a screen for CRT nonresponders in patients with HF and LBBB.

\section{Abbreviations \\ 3D: 3-Dimensional; C: Capacitance; CO: Cardiac output; CRT: Cardiac resyn- chronization therapy; CT: Computed tomography; DBP: Diastolic blood pressure; ECG: Electrocardiography; HF: Heart failure; HR: Heart rate; LBBB: Left bundle branch block; LV: Left ventricle; LVEDV: Left ventricular end-diastolic volume; LVEF: Left ventricular ejection fracture; LVESV: Left ventricular end- systolic volume; MR: Magnetic resonance; R: resistance; RV: Right ventricle; SBP: Systolic blood pressure.}

\section{Acknowledgements}

Not applicable.

\section{Authors' contributions}

$\mathrm{MH}$ and MCP contributed to the concept and design of the work, acquisition and interpretation of data for the work, and drafting of the manuscript. JSU and EBS contributed to the concept and design of the work, acquisition and 
interpretation of data for the work, and critical revision of the manuscript. CJL, JO, HTY, THK, BJ, HNP, SMK, and MHL contributed to the design of the work, acquisition and interpretation of data for the work, and critical revision of the manuscript. All authors approved the final version to be published and agree to be accountable for all aspects of the work in ensuring that questions related to the accuracy or integrity of any part of the work are appropriately investigated and resolved. All authors read and approved the final manuscript.

\section{Funding}

This research was supported by a grant from the Korean Heart Rhythm Society (2017-31-0602), the Korean Cardiac Research Foundation (201903-01), and the National Research Foundation of Korea (2020R1G1A1014558).

\section{Availability of supporting data}

The datasets are available from the corresponding author on reasonable request.

\section{Declarations}

\section{Ethical approval and consent to participate}

The study protocol adhered to the Declaration of Helsinki and was approved by the institutional review board (1-2019-0077 and 1-2020-0008).

\section{Consent for publication}

Written informed consent for publication of their clinical data was waived owing to the study's retrospective nature and the absence of patient identification data presented.

\section{Competing interests}

The authors declare no conflict of interests.

\section{Author details}

${ }^{1}$ Al Medic Inc., Seoul, Republic of Korea. ${ }^{2}$ Department of Cardiology, Yongin Severance Hospital, Yonsei University College of Medicine, 363 Dongbaekjukjeon-daero, Giheung-gu, Yongin-si, Gyeonggi-do 16995, Republic of Korea. ${ }^{3}$ Department of Mechanical and Biomedical Engineering, Kangwon National University, Chuncheon, Gangwon-do, Republic of Korea. ${ }^{4}$ Division of Cardiology, Department of Internal Medicine, Severance Hospital, Yonsei University College of Medicine, Seoul, Republic of Korea.

Received: 13 June 2021 Accepted: 12 October 2021

Published online: 05 January 2022

\section{References}

1. Rickard J, Michtalik H, Sharma R, Berger Z, lyoha E, Green AR, et al. Predictors of response to cardiac resynchronization therapy: a systematic review. Int J Cardiol. 2016;225:345-52.

2. Katbeh A, Van Camp G, Barbato E, Galderisi M, Trimarco B, Bartunek J, et al. Cardiac resynchronization therapy optimization: a comprehensive approach. Cardiology. 2019;142(2):116-28.

3. Daubert C, Behar N, Martins RP, Mabo P, Leclercq C. Avoiding nonresponders to cardiac resynchronization therapy: a practical guide. Eur Heart J. 2017;38:1463-72.

4. Trayanova NA, Constantino J, Gurev V. Electromechanical models of the ventricles. Am J Physiol Heart Circ Physiol. 2011;301:H279-86.

5. Sugiura S, Washio T, Hatano A, Okada J, Watanabe H, Hisada T. Multiscale simulations of cardiac electrophysiology and mechanics using the University of Tokyo heart simulator. Prog Biophys Mol Biol. 2012;110:380-9.

6. Kim YT, Lee JS, Youn CH, Choi JS, Shim EB. An integrative model of the cardiovascular system coupling heart cellular mechanics with arterial network hemodynamics. J Korean Med Sci. 2013;28:1161-8.

7. Lee KE, Kim KT, Lee JH, Jung S, Kim JH, Shim EB. Computational analysis of the electromechanical performance of mitral valve cerclage annuloplasty using a patient-specific ventricular model. Korean J Physiol Pharmacol. 2019;23:63-70.

8. Im UB, Kwon SS, Kim K, Lee YH, Park YK, Youn CH, et al. Theoretical analysis of the magnetocardiographic pattern for reentry wave propagation in a three-dimensional human heart model. Prog Biophy Mol Biol. 2008;96:339-56.

9. Shim EB, Amano A, Takahata T, Shimayoshi T, Noma A. The cross-bridge dynamics during ventricular contraction predicted by coupling the cardiac cell model with a circulation model. J Physiol Sci. 2007;57:275-85.

10. Lim KM, Hong SB, Lee BK, Shim EB, Trayanova N. Computational analysis of the effect of valvular regurgitation on ventricular mechanics using a 3D electromechanics model. J Physiol Sci. 2015;65:159-64.

11. Lim KM, Jeon JW, Gyeong MS, Hong SB, Ko BH, Bae SK, et al. Patientspecific identification of optimal ubiquitous electrocardiogram (U-ECG) placement using a three-dimensional model of cardiac electrophysiology. IEEE Trans Biomed Eng. 2013;60:245-9.

12. Uhm JS, Oh J, Cho IJ, Park M, Kim IS, Jin MN, et al. Left ventricular endsystolic volume can predict 1-year hierarchical clinical composite end point in patients with cardiac resynchronization therapy. Yonsei Med J. 2019;60:48-55.

13. Cho IJ, Uhm JS, Oh J, Nam JH, Yu HT, Kim T, et al. Left ventricular response after cardiac resynchronization therapy is related to early left atrial volume reduction. Korean J Intern Med. 2020;35:1125-35.

14. Strauss DG, Selvester RH, Wagner GS. Defining left bundle branch block in the era of cardiac resynchronization therapy. Am J Cardiol. 2011;107:927-34.

15. Lee AWC, Costa CM, Strocchi M, Rinaldi CA, Niederer SA. Computational modeling for cardiac resynchronization therapy. J Cardiovasc Transl Res. 2018;11:92-108.

16. Derval N, Steendijk P, Gula LJ, Deplagne A, Laborderie J, Sacher F, et al. Optimizing hemodynamics in heart failure patients by systematic screening of left ventricular pacing sites: the lateral left ventricular wall and the coronary sinus are rarely the best sites. J Am Coll Cardiol. 2010;55:566-75.

17. Spragg DD, Dong J, Fetics BJ, Helm R, Marine JE, Cheng A, et al. Optimal left ventricular endocardial pacing sites for cardiac resynchronization therapy in patients with ischemic cardiomyopathy. J Am Coll Cardiol. 2010;56:774-81.

18. Isotani A, Yoneda K, Iwamura T, Watanabe M, Okada Jl, Washio T, et al. Patient-specific heart simulation can identify non-responders to cardiac resynchronization therapy. Heart Vessels. 2020;35:1135-47.

19. Okada JI, Washio T, Nakagawa M, Watanabe M, Kadooka Y, Kariya T, et al. Multi-scale, tailor-made heart simulation can predict the effect of cardiac resynchronization therapy. J Mol Cell Cardiol. 2017;108:17-23.

20. Okada JI, Washio T, Sugiura S, Hisada T. Clinical and pharmacological application of multiscale multiphysics heart simulator. UT-Heart Korean J Physiol Pharmacol. 2019;23:295-303.

\section{Publisher's Note}

Springer Nature remains neutral with regard to jurisdictional claims in published maps and institutional affiliations. 